\title{
ARHGEF1 deficiency reveals Ga13-associated GPCRs are critical regulators of human lymphocyte function
}

\author{
Divij Mathew, Kimberly N. Kremer, and Raul M. Torres
}

Department of Immunology and Microbiology, University of Colorado School of Medicine, Aurora, Colorado, USA

\begin{abstract}
Primary antibody deficiencies are the most common immunodeficiencies in humans; however, identification of the underlying genetic and biochemical basis for these diseases is often difficult, given that these deficiencies typically involve complex genetic etiologies. In this issue of the JCI, Bouafia et al. performed whole-exome sequencing on a pair of siblings with primary antibody deficiencies and identified genetic mutations that result in a deficiency of ARHGEF1, a hematopoietic intracellular signaling molecule that transmits signals from GPCRs. ARHCEF1-deficient lymphocytes from the affected siblings exhibited important functional deficits that indicate that loss of ARHGEF1 accounts for the observed primary antibody deficiency, which manifests in an inability to mount antibody responses to vaccines and pathogens. Thus, this report demonstrates an important role for ARHCEF1 in GPCR signal transduction required for appropriate adaptive immune responses in humans.
\end{abstract}

\section{Introduction}

Primary antibody deficiencies (PADs) include a broad spectrum of disorders that range from the absence of B lymphocytes and serum Ig to normal serum Ig isotypes and concentrations, but aberrant antibody responses $(1,2)$. The identification of genetic mutations that underlie and lead to PADs, and in particular X-linked agammaglobulinemias, has benefited from the analyses of natural and engineered genetic mutations in mouse models that lead to impaired B cell development $(3,4)$. However, in the current era of -omics (genomics, proteomics, lipidomics, and metabalomics), it is now possible to identify genetic lesions that underlie symptoms in affected patients and subsequently characterize the manifestation of these mutations on lymphocyte function. In this issue, Bouafia et al. (5) report on their performance and analysis of whole-exome sequencing of siblings presenting with recurrent respiratory tract infection, bronchiectasis, and an inability to mount antigen-specific antibody responses. These analyses led to the identification of two distinct ARHGEF1 mutant alleles that, together as compound heterozygous mutations, resulted in an ARHGEF1 deficiency.

\section{ARHGEF1}

ARHGEF1 was first identified over two decades ago $(6,7)$ as an intracellular signaling molecule with two biochemically defined functions (Figure 1). The first activity is contained within a regulator of G-protein signaling (RGS) domain that functions to accelerate the inherent GTPase activity of $\mathrm{G} \alpha$ heterotrimeric G-protein subunits (8). ARHGEF1 RGS activity is specific for $G \alpha 12$ and $G \alpha 13$ subunits, and thus ARHGEF1 attenuates signaling from Ga12/13-associated GPCRs (9). The second activity is contained within a tandem Dbl- and pleckstrinhomology (DH-PH) domain that harbors GEF activity specific for the small molecular weight RhoA GTPase (10). Although ARHGEF1 RGS activity acts on

Related Article: p. 1047

Conflict of interest: The authors have declared that no conflict of interest exists.

Reference information: / Clin Invest. 2019;129(3):965-968. https://doi.org/10.1172/JCI125893.

both $G \alpha 12$ and $G \alpha 13$, only the association with active Ga13 stimulates its RhoA GEF activity (10). Thus, based on these biochemically defined activities, ARHGEF1 functions to terminate GPCR signaling from $\mathrm{G} \alpha 13$-associated receptors and subsequently activate RhoA, an important regulator of the actin cytoskeleton with pleiotropic functions.

ARHGEF1 is predominantly expressed in cells of hematopoietic origin, and as GPCRs are well characterized as regulating diverse activities in immune cells, such as trafficking, differentiation, survival, and proliferation, several Arhgef1 ${ }^{-/-}$mouse mutants were independently generated (11-13) to investigate how Ga12/13-associated GPCRs use ARHGEF1 to regulate these functions. The aggregate results from analyses of ARHGEF1 mouse mutants revealed an important role for ARHGEF1 in the development of mature B cell populations (e.g., marginal zone [MZ] B cells) and B lymphocyte migration that, when disrupted, ultimately resulted in markedly impaired antigen-specific antibody responses to both $\mathrm{T}$ cell-dependent and $\mathrm{T}$ cell-independent antigens $(11,12)$. Although the precise Ga12/13-associated GPCR or GPCRs responsible for the development and function of MZ B cells are not yet fully identified, the results from these mouse models indicate that a Ga13-associated GPCR/ARHGEF1 signaling pathway is important for MZ B cell-mediated humoral immunity. Further studies are necessary to identify which GPCRs contribute to the development of humoral immunity and whether these GPCRs function to activate specific transcriptional programs, position MZ B cells in distinct anatomical locations, or promote specific cell-cell interactions that are important for appropriate differentiation.

Clinical manifestations of an ARHGEF1 deficiency

Bouafia et al. (5) focus on a set of ARHGEF1-deficient siblings who presented 


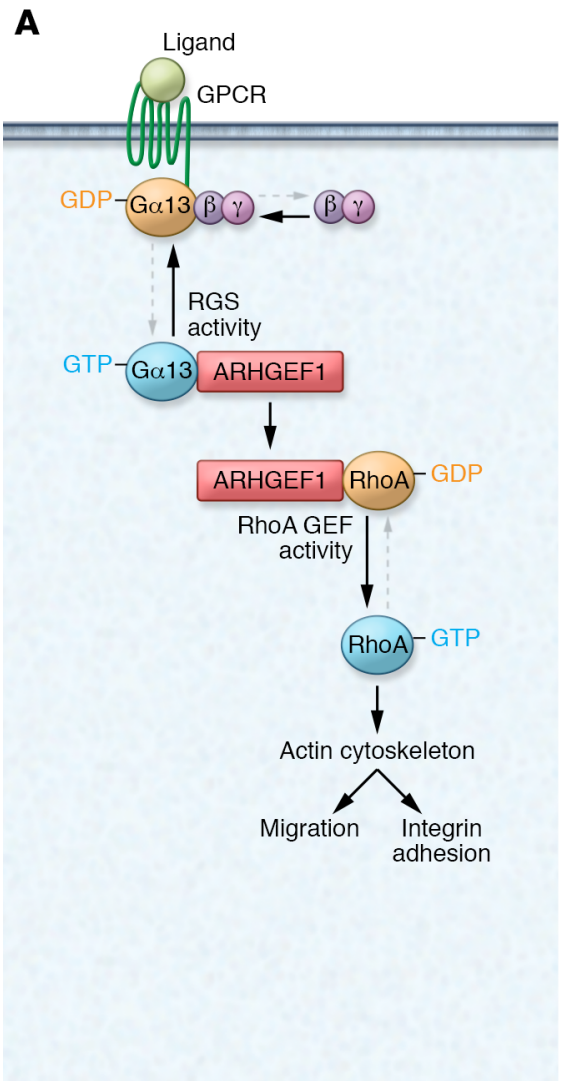

early in childhood with recurrent upper and lower respiratory tract infections, depressed serum concentrations of IgG, and, in one sibling, reduced $\operatorname{IgA}$ and $\operatorname{IgE}$ levels. Notably, neither sibling harbored measurable antibody titers against protein and viral vaccines or Streptococcus pneumonia, indicating defective antigenspecific antibody responses to both protein antigens and pneumococcal polysaccharides. Work from rodent models has established that protein antigens elicit antibody responses from $\mathrm{B}$ cells that are dependent on $\mathrm{T}$ cell help (TD antibody responses), whereas antibody responses against capsular polysaccharides can be elicited independently of $\mathrm{T}$ cells (TI antibody responses) (14). Indeed, consistent with the observed defective antibody responses, analysis of peripheral blood from the affected siblings revealed a marked reduction in the presence of $B$ lymphocytes, specifically MZ and memory B cell populations, which was similar to previous findings in ARHGEF1-deficient mice. Finally, although T cell frequencies were within the normal range, peripheral blood memory $\mathrm{CD}^{+} \mathrm{T}$ cells were severely reduced in frequency.

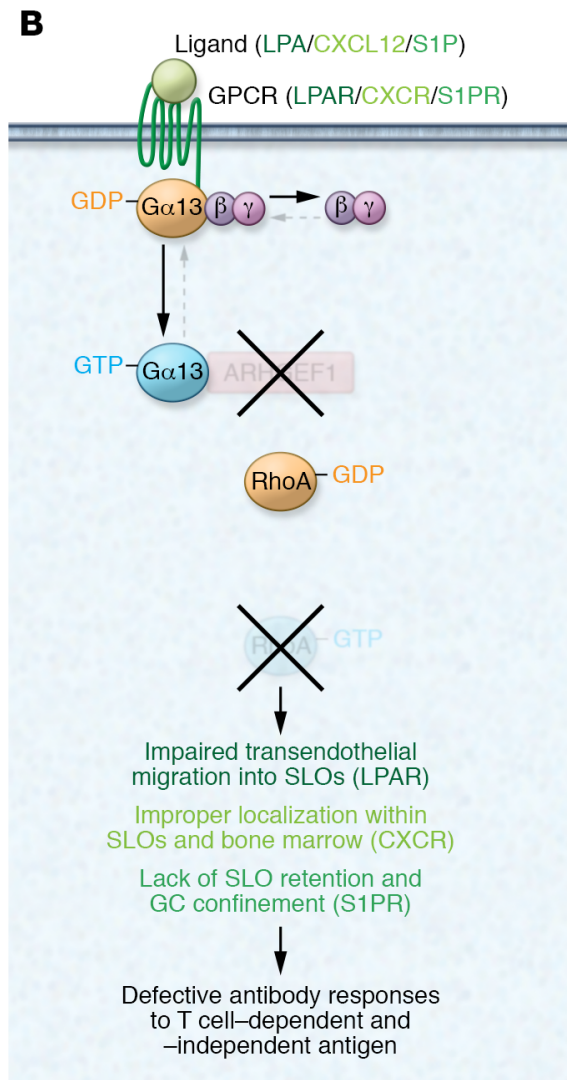

\section{ARHGEF1 signaling functions important for humoral immunity}

The presence of B lymphocytes was markedly diminished in the peripheral blood of both ARHGEF1-deficient siblings. Consequently, to document signaling abnormalities in ARHGEF1-deficienct lymphocytes isolated from affected siblings, Bouafia et al. (5) relied largely on primary T cells and $\mathrm{T}$ cell blasts in most of their in vitro experiments; however, transformed mutant B cells had analogous results in some experiments. More precisely, the authors treated ARHGEF1-deficient lymphocytes with agonist ligands for Ga13-associated GPCRs, including sphingosine-1-phosphate (S1P), lysophosphatidic acid (LPA), thromboxane, and CXCL12, and retrovirally introduced GNA13 (encoding Ga13) and ARHGEF1 transgenes into mutant $\mathrm{B}$ cells to convincingly demonstrate that loss of ARHGEF1 leads to impaired Ga13-associated GPCR signaling. Loss of ARHGEF1 in affected lymphocytes resulted in diminished RhoA activation, reduced actin polymerization, an inability to suppress AKT activity, and impaired migration on integrin ligands. These functional defi-
Figure 1. ARHGEF1 is an intracellular signaling effector that activates RhoA in response to G $\alpha 12 / 13$-associated GPCR signaling. (A) ARHGEF1 RGS activity terminates G $\alpha 12 / 13$-associated GPCR signaling and stimulates ARHGEF1 RhoA GEF activity. Active GTP-bound RhoA controls actin cytoskeleton and is required for appropriate integrin adhesion and migration. (B) ARHCEF1-deficient lymphocytes would not efficiently terminate GPCR signaling nor activate RhoA, resulting in impaired $G \alpha 12 / 13$-associated GPCR signaling through S1P, chemokine, and LPA GPCRs. As a result, ARHGEF1-deficient lymphocytes display impaired adhesion and trafficking, ultimately leading to aberrant localization in SLOs and germinal centers and defective antigen-specific antibody responses.

cits are reminiscent of previous analyses of engineered mouse mutants, including Arhgef1 $1^{-/-}$and S1pr2 ${ }^{-/-}$mice $\left(11^{-13}, 15\right)$, and are consistent with characterized Ga13associated GPCR signaling pathways, such as CXCR4 and LPARs/S1PRs (1618). Notably, CXCR4, like all chemokine receptors, signals chemotactic migration via pertussis toxin-sensitive Gai-associated heterotrimeric proteins. However, CXCR4 expressed by certain cell types has been shown to use Ga13-associated signaling to Rho to contribute to cell migration, and in T cells, a CXCR4/Ga13/RhoA axis has been demonstrated to be important in recycling CXCR4 back to the cell surface after ligation with CXCL12 (16-18). Thus, the immunological deficits of these patients are likely due to the impairment of ARHGEF1-dependent signaling pathways downstream of these GPCRs.

\section{ARHGEF1 deficiency leads to a PAD}

In rodent animal models, the antibody response to protein and polysaccharide antigens has been characterized as stratifying into TD and TI antibody responses, respectively. Furthermore, different B 
cell populations are considered responsible for mounting these TD and TI antibody responses, and this division of $\mathrm{B}$ cell labor in humoral immunity is supported by human studies (14). Thus, the antibody response to TD antigens is elicited from the major population of naive $\operatorname{Ig} \mathrm{M}^{+} \operatorname{IgD}{ }^{+} \mathrm{CD} 27^{-}$ $\mathrm{B}$ cells in humans, whereas a human $\operatorname{IgM}^{+} \operatorname{IgD}{ }^{+} \mathrm{CD} 27^{+}$subpopulation, analogous to murine MZ B cells (19), has been reported as mounting TI antibody responses to encapsulated bacteria, such as S. pneumoniae, Neisseria meningitidis, and Haemophilus influenzae (20-22). Although B cell frequencies were markedly diminished in both siblings, the MZ and memory B cell populations were more severely affected and likely contributed to the impaired TI antibody responses to $S$. pneumoniae and reduced Ig serum concentrations. Thus, in consideration of the findings of Bouafia et al. (5) and previously characterized mouse mutants, it is clear that ARHGEF1 is not only required for the appropriate development of MZ B cells, but is also required for their ability to mount antibody responses.

To mount TD antibody responses, B cells must physically interact with $\mathrm{T}$ cells that are specific for the same antigen. This interaction is accomplished via the migration of $\mathrm{B}$ and $\mathrm{T}$ cells to the same area of secondary lymphoid organs (SLOs) after recognition of the same cognate antigen. Subsequent to a productive T-B cell interaction, activated B cells migrate into B cell follicles, form germinal centers - specialized structures where B cells undergo extensive proliferation, mutate their antigen receptors to increase antigen affinity, and undergo Ig class switch recombination to produce diverse Ig isotypes - and ultimately exit as either memory B cells or antibody-secreting plasma cells. Importantly, several GPCRs, including CXCR4, CXCR5, EBI2, S1P chemokine, and lipid GPCRs, have been shown to regulate $B$ cell trafficking to and retention within the germinal center (15). In a related finding, Bouafia et al. documented a paucity of B cells in germinal centers from available lymph node biopsies from one of the affected siblings, indicating that, as shown in mouse models, ARHGEF1 signaling downstream of GPCRs in human germinal centers promotes localization and differentiation. Specifically, S1P2 receptor signaling via ARHGEF1 in mice confines responding $\mathrm{B}$ cells within the germinal center, where high-affinity switched Ig isotypes are generated and from where memory B cells and plasma cells emerge. Thus, in ARHGEF1-deficient individuals, the depressed IgG serum levels and absence of memory B cells would be in accordance with described ARHGEF1 germinal center B cell functions (15). Finally, based on the virtual absence of MZ and memory B cells in the affected siblings coupled with previous analyses of mouse mutants, it stands to reason that an ARHGEF1 deficiency in B cells leads to defective humoral immunity (Figure 1). However, considerable data are presented that would also suggest that loss of ARHGEF1 in $\mathrm{T}$ cells likely contributes to the aberrant antibody response to $\mathrm{T}$ cell-dependent protein and viral antigens.

\section{Conclusions}

Given the typical complex genetic etiology for PADs, it is noteworthy that Bouafia et al. (5) provide compelling evidence that an ARHGEF1 deficiency leads to significant impairment in B and T lymphocyte differentiation, migration, and GPCR-mediated signaling that appears to account for the observed PAD. The identification of ARHGEF1 deficiency that can lead to a PAD clearly establishes a critical role for ARHGEF1, and by extension Ga12/13-associated GPCR signaling, in regulating lymphocyte development and function. Based on the data presented by Bouafia et al. and previous findings on ARHGEF1, Ga12/13, SIPR2, and CXCR4 mouse mutants and characterized ARHGEF1 signaling pathways, we have outlined how an ARHGEF1 deficiency might lead to defective antigen-specific antibody responses (Figure 1). Thus, Bouafia, et al. provide valuable insight into how loss of a single protein can alter the signaling of many different GPCRs to impair humoral immunity by altering B cell development and function. The use of animal models in conjunction with-omics in the future will likely be an important combination to enhance the success of individualized medicine.

\section{Acknowledgments}

Work in our lab has been funded in part by NIH R01 AI052157 and AI136534, the Cancer League of Colorado (to RMT), and T32 AI007405 (to DM).
Address correspondence to: Raul M. Torres, 12800 E.19th Avenue, MS 8333, Aurora, Colorado 80045, USA. Phone: 303.724.8669;

Email: raul.torres@ucdenver.edu.

1. Ballow M. Primary immunodeficiency disorders: antibody deficiency. J Allergy Clin Immunol. 2002;109(4):581-591.

2. Durandy A, Kracker S, Fischer A. Primary antibody deficiencies. Nat Rev Immunol. 2013;13(7):519-533.

3. Conley ME, Rohrer J, Rapalus L, Boylin EC, Minegishi Y. Defects in early B-cell development: comparing the consequences of abnormalities in pre-BCR signaling in the human and the mouse. Immunol Rev. 2000;178:75-90.

4. Schiff C, Lemmers B, Deville A, Fougereau M, Meffre E. Autosomal primary immunodeficiencies affecting human bone marrow B-cell differentiation. Immunol Rev. 2000;178:91-98.

5. Bouafia A, et al. Loss of ARHGEF1 causes a human primary antibody deficiency. JClin Invest. 2019;129(3):1047-1060.

6. Whitehead IP, Khosravi-Far R, Kirk H, TrigoGonzalez G, Der CJ, Kay R. Expression cloning of lsc, a novel oncogene with structural similarities to the Dbl family of guanine nucleotide exchange factors. J Biol Chem. 1996;271(31):18643-18650.

7. Hart MJ, et al. Identification of a novel guanine nucleotide exchange factor for the Rho GTPase. J Biol Chem. 1996;271(41):25452-25458.

8. Hollinger S, Hepler JR. Cellular regulation of RGS proteins: modulators and integrators of $\mathrm{G}$ protein signaling. Pharmacol Rev. 2002;54(3):527-559.

9. Kozasa T, et al. p115 RhoGEF, a GTPase activating protein for Galpha12 and Galpha13. Science. 1998;280(5372):2109-2111.

10. Hart MJ, et al. Direct stimulation of the guanine nucleotide exchange activity of p115 RhoGEF by Galpha13. Science. 1998;280(5372):2112-2114.

11. Girkontaite I, et al. Lsc is required for marginal zone B cells, regulation of lymphocyte motility and immune responses. Nat Immunol. 2001;2(9):855-862.

12. Rubtsov A, Strauch P, Digiacomo A, Hu J, Pelanda R, Torres RM. Lsc regulates marginal-zone $B$ cell migration and adhesion and is required for the IgM T-dependent antibody response. Immunity. 2005;23(5):527-538.

13. Francis SA, Shen X, Young JB, Kaul P, Lerner DJ. Rho GEF Lsc is required for normal polarization, migration, and adhesion of formyl-peptide-stimulated neutrophils. Blood. 2006;107(4):1627-1635.

14. Swanson CL, Pelanda R, Torres RM. Division of labor during primary humoral immunity. Immunol Res. 2013;55(1-3):277-286.

15. Green JA, Cyster JG. S1PR2 links germinal center confinement and growth regulation. Immunol Rev. 2012;247(1):36-51.

16. Tan W, Martin D, Gutkind JS. The Galpha13-Rho signaling axis is required for SDF-1-induced migration through CXCR4. J Biol Chem. 2006;281(51):39542-39549.

17. Kumar A, Kremer KN, Dominguez D, Tadi M, Hedin KE. Ga13 and Rho mediate endosomal 
trafficking of CXCR4 into Rab11+ vesicles upon stromal cell-derived factor-1 stimulation. JImmunol. 2011;186(2):951-958.

18. Yagi H, et al. A synthetic biology approach reveals a CXCR4-G13-Rho signaling axis driving transendothelial migration of metastatic breast cancer cells. Sci Signal. 2011;4(191):ra60.

19. Weller S, et al. Human blood IgM "memory" B cells are circulating splenic marginal zone B cells harboring a prediversified immunoglobulin repertoire. Blood. 2004;104(12):3647-3654.

20. Kruetzmann S, et al. Human immunoglobulin M memory B cells controlling Streptococcus pneumoniae infections are generated in the spleen. JExp Med. 2003;197(7):939-945.

21. Carsetti R, et al. The loss of IgM memory B cells correlates with clinical disease in common variable immunodeficiency. JAllergy Clin Immunol. 2005;115(2):412-417.

22. Verbinnen B, Covens K, Moens L, Meyts I, Bossuyt X. Human CD20+CD43+CD27+CD5$\mathrm{B}$ cells generate antibodies to capsular polysaccharides of Streptococcus pneumoniae. J Allergy Clin Immunol. 2012;130(1):272-275. 